\title{
Robust Output Feedback MPC: An Interval-Observer Approach
}

\author{
Alex Reis de Souza, Denis Efimov, Tarek Raïssi, Xubin Ping
}

\begin{abstract}
In this work, we address the problem of outputfeedback Model Predictive Control (MPC) of constrained, linear, discrete-time systems corrupted by additive perturbations on both state and output. The use of estimated variables in MPC is challenging and computationally expensive due to constraint satisfaction. To overcome this issue, the proposed approach incorporates interval observers on the MPC scheme to cope with uncertainty, leading to a novel, simple and very intuitive methodology providing robust constraint satisfaction with reduced computational complexity.
\end{abstract}

\section{INTRODUCTION}

MPC is a feedback control technique based on the iterative solution of an optimization problem [1]. Using the current information about the states, this optimization problem relies on (model-based) prediction of the future behavior of the system, aiming to find the best control sequence that accomplishes the control task. This is a very attractive methodology since it allows the consideration of a multi-variable system, as well as constraint handling on both state and control. Indeed, even for linear systems, constrained control problems are often difficult or impossible to tackle with classical feedback methods [1].

However, two important issues arise in this methodology: (i) full-state measurement is not always available, leading to the necessity of state estimation, and (ii) measurements and the model available for prediction are often uncertain (for instance, due to unmodelled dynamics, exogenous disturbances, and measurement noises). This fact represents a challenging issue, especially when the system to be controlled is constrained [2], leading to the need for MPC schemes with robust constraint satisfaction guarantees, which are computationally complex. Such robust MPC schemes have been studied over the last decades, being tackled by different approaches such as set-membership estimation [3], min-max optimization [4], and tube-based approaches [5], [6].

This work is partially supported by IPL CoSY, by the Government of Russian Federation (Grant 08-08), and by the Ministry of Science and Higher Education of Russian Federation, passport of goszadanie no. 2019-0898

A. Reis de Souza and D. Efimov are with Inria, Univ. Lille, CNRS, UMR 9189 - CRIStAL, F-59000 Lille, France.

D. Efimov is with ITMO University, 49 av. Kronverkskiy, 197101 Saint Petersburg, Russia.

Tarek Raïssi is with Conservatoire National des Arts et Métiers (CNAM), Cedric 292, Rue St-Martin, 75141 Paris, France.

Xubin Ping is with School of Electro-Mechanical Engineering, Xidian University, China.
Being a special class of set-membership estimators, an interval observer (IO) [7] uses input-output information and interval bounds of the model uncertainties to evaluate the set of admissible values for the state (i.e., interval) at each instant of time. The width of these intervals, which is to be minimized by a proper tuning of the observer, is then proportional to the magnitude of the uncertainty. The design of IOs has been exhaustively studied, and the literature reports results on linear [8], linear parameter-varying [9], nonlinear [10], discrete-time [11], as well as on time-delay systems [12]. Recently an interval predictor (IP), sometimes also called framer, was presented in [13], [14] for systems in continuous time.

In this paper, we consider the problem of output-feedback design for constrained, linear, discrete-time systems subject to state and measurement perturbations. The main challenge in this scenario relies on guaranteeing that states and input constraints are robustly satisfied despite of all uncertainties. In the proposed MPC scheme, we will show that interval observers and predictors offer a very intuitive and computationally inexpensive way to cope with these uncertainties, provided that some conditions on non-negativity, formulated in terms of linear matrix inequalities, can be verified. It is worth noticing that the proposed method differs from the literature (such as [3]) due to its observer form and guaranteed estimation features.

The goal of this paper is to demonstrate the advantages of using IO and IP on a simple case of linear systems with additive disturbances, while leaving the possible extensions to more complex classes of systems (e.g., linear parametervarying ones) to future researches.

The outline of this paper is as follows. In Section II the problem statement is given. Designs of IO and IP are considered in Section III. Application of IO and IP for MPC is studied in Section IV. Comparison of the proposed interval based MPC framework and a conventional solution is presented in Section V.

\section{NOTATION:}

- The sets of real and integer numbers are defined by $\mathbb{R}$ and $\mathbb{Z}$, respectively, then $|\cdot|$ represents the absolute value for an element of these sets; $\mathbb{R}_{+}=\{s \in \mathbb{R}: s \geq 0\}$ and $\mathbb{Z}_{+}=\mathbb{Z} \cap \mathbb{R}_{+}$. The Euclidean norm of a vector $x \in \mathbb{R}^{n}$ is denoted by $\|x\|$.

- A matrix $A \in \mathbb{R}^{n \times n}$ is said to be nonnegative if all of its elements are nonnegative. A matrix $A \in \mathbb{R}^{n \times n}$ is said 
to be Schur stable if all of its eigenvalues have absolute value less than one. The identity matrix of dimension $n \times n$ is denoted by $I_{n}$.

- For a bounded function $x: \mathbb{Z}_{+} \rightarrow \mathbb{R}^{n}$, we use the convention $x_{k}=x(k)$ for any $k \in \mathbb{Z}_{+}$and denote $|x|_{\infty}=\sup _{k \in \mathbb{Z}_{+}}\left\|x_{k}\right\|$. Furthermore, we define as $\mathcal{L}_{\infty}^{n}$ the set of all such functions with $|x|_{\infty}<\infty$.

- Let $x_{1}, x_{2} \in \mathbb{R}^{n}$ be two vectors and $A_{1}, A_{2} \in \mathbb{R}^{n \times n}$ be two matrices, then the relations $x_{1} \leq x_{2}$ and $A_{1} \leq A_{2}$ are to be understood component-wise. For a matrix $A$ we define $A^{+}=\max \{0, A\}, A^{-}=A^{+}-A$ (similarly for vectors), and also denote the matrix of absolute values of all elements by $|A|=A^{+}+A^{-}$. Furthermore, for a symmetric matrix $A \in \mathbb{R}^{n \times n}$ the relation $A \prec 0$ (resp. $A \succeq 0$ ) means that it is negative (resp. positive semi-) definite.

\section{Problem Statement}

Consider the following uncertain, linear, discrete-time system given for $k \in \mathbb{Z}_{+}$by

$$
\begin{aligned}
x_{k+1} & =A x_{k}+B u_{k}+w_{k}, \\
y_{k} & =C x_{k}+v_{k},
\end{aligned}
$$

where $x_{k} \in \mathbb{R}^{n}$ is the state vector, $u_{k} \in \mathbb{R}^{m}$ is the input vector and $y_{k} \in \mathbb{R}^{p}$ is the vector of available measurements; the signals $w \in \mathcal{L}_{\infty}^{n}$ and $v \in \mathcal{L}_{\infty}^{p}$ are, respectively, process and measurement disturbances; the constant matrices $A \in$ $\mathbb{R}^{n \times n}, B \in \mathbb{R}^{n \times m}$ and $C \in \mathbb{R}^{p \times n}$ are known. The following hypotheses are imposed:

Assumption 1. Initial conditions of (1) are bounded such as $\underline{x}_{0} \leq x_{0} \leq \bar{x}_{0}$, for some known $\underline{x}_{0}, \bar{x}_{0} \in \mathbb{R}^{n}$.

Assumption 2. The additive perturbations $w_{k} \in\left[\underline{w}_{k}, \bar{w}_{k}\right]$ and $v_{k} \in\left[\underline{v}_{k}, \bar{v}_{k}\right]$ for all $k \in \mathbb{Z}_{+}$, where $\underline{w}, \bar{w} \in \mathcal{L}_{\infty}^{n}$ and $\underline{v}, \bar{v} \in \mathcal{L}_{\infty}^{p}$ are known signals.

Thus, the three sources of uncertainty in (1), $x_{0}, w_{k}$ and $v_{k}$, are enclosed in given intervals, $\left[\underline{x}_{0}, \bar{x}_{0}\right],\left[\underline{w}_{k}, \bar{w}_{k}\right]$ and $\left[\underline{v}_{k}, \bar{v}_{k}\right]$, respectively, which is a usual case in the theory of design of IOs [15].

Furthermore, we suppose that for all $k \in \mathbb{Z}_{+}$both, states $x_{k}$ and controls $u_{k}$, have to be constrained by bounded sets $\mathbb{X} \subset \mathbb{R}^{n}$ and $\mathbb{U} \subset \mathbb{R}^{m}$, respectively (which can be, for instance, presented by bounded intervals $\mathbb{X}=[\chi, \bar{\chi}]$ and $\mathbb{U}=[\underline{u}, \bar{u}]$, considering $\chi, \bar{\chi} \in \mathbb{R}^{n}$ and $\underline{u}, \bar{u} \in \overline{\mathbb{R}}^{m}$ to be known bounds); $0 \in \mathbb{X}$ and $0 \in \mathbb{U}$.

Problem. Let $\left[\underline{x}_{0}, \bar{x}_{0}\right] \subset \mathbb{X}$ and assumptions 1,2 be satisfied. Design an output-feedback controller stabilizing the system (1) in a terminal set $\mathbb{X}_{f} \subset \mathbb{R}^{n}$ (a vicinity of the origin) and providing

$$
x_{k} \in \mathbb{X}, u_{k} \in \mathbb{U} \quad \forall k \in \mathbb{Z}_{+} .
$$

Following the paradigm of MPC [1], [2], the solution of this problem can be decomposed on three stages: $a$ ) design of an observer, $b$ ) design of a predictor, and $c$ ) design of a control. Due to imposed interval structure of uncertainty, in this work we will design an IO and an IP to solve $a$ ) and $b$ ) in the next section, and further we will adapt the conventional MPC framework for using the interval estimation and prediction algorithms to solve $c$ ).

As it will be demonstrated, the main advantage of using the interval algorithms is their weak computational complexity comparing to conventional solutions [5], [6], provided that some additional restrictions on positivity of estimation/prediction errors can be satisfied by design of the gains.

\section{INTERVAL ESTIMATION AND PREDICTION}

The objective is to design estimators that, while having an observer form, provide guaranteed information on the setmembership of the system states. The role of the IO and the IP as well distinguished in two scenarios: the IO, using output injection, will be responsible for estimating the interval at each instant of time $k \in \mathbb{Z}_{+}$, while the IP will be responsible for estimating intervals for $k+1, \ldots, k+N$, where $N>0$ is the prediction horizon of the MPC. In both cases, estimates $\underline{x}_{k}, \bar{x}_{k} \in \mathbb{R}^{n}$ are computed such that the relation

$$
\underline{x}_{k} \leq x_{k} \leq \bar{x}_{k} \quad \forall k \in \mathbb{Z}_{+}
$$

holds under assumptions 1 and 2 .

We will use the following results in the sequel:

Lemma 1. [16] Let $A$ be a matrix of proper dimensions and $x, \underline{x}, \bar{x} \in \mathbb{R}^{n}$ be such that $\underline{x} \leq x \leq \bar{x}$, then

$$
A^{+} \underline{x}-A^{-} \bar{x} \leq A x \leq A^{+} \bar{x}-A^{-} \underline{x} .
$$

Lemma 2. [15] For $A \in \mathbb{R}_{+}^{n \times n}$ the system

$$
x_{k+1}=A x_{k}+\omega_{k}, \omega: \mathbb{Z}_{+} \rightarrow \mathbb{R}_{+}^{n}, \omega \in \mathcal{L}_{\infty}^{n}, k \in \mathbb{Z}_{+},
$$

has a nonnegative solution $x_{k} \in \mathbb{R}_{+}^{n}$ for all $k \in \mathbb{Z}_{+}$provided that $x_{0} \geq 0$.

A system as in above lemma is called cooperative (monotone) or nonnegative.

Lemma 3. [17] A matrix $A \in \mathbb{R}_{+}^{n \times n}$ is Schur stable iff there exists a diagonal matrix $P \in \mathbb{R}^{n \times n}, P>0$ such that $A^{\top} P A-P \prec 0$.

\section{A. Interval Observer}

First, let us consider the possibility of using output-injection to design such an estimator. To this end (1) can be rewritten as

$$
x_{k+1}=(A-L C) x_{k}+B u_{k}+L y_{k}-L v_{k}+w_{k}
$$

for any $L \in \mathbb{R}^{n \times p}$. Following [11], replacing in (4) the uncertain terms (i.e., $w_{k}-L v_{k}$ ) by their interval bounds with the use of (3), we propose the following IO:

$$
\begin{aligned}
& \bar{x}_{k+1}=(A-L C) \bar{x}_{k}+B u_{k}+L y_{k}+\bar{w}_{k}-L^{+} \underline{v}_{k}+L^{-} \bar{v}_{k} \\
& \underline{x}_{k+1}=(A-L C) \underline{x}_{k}+B u_{k}+L y_{k}+\underline{w}_{k}-L^{+} \bar{v}_{k}+L^{-} \underline{v}_{k}
\end{aligned},
$$


where $L \in \mathbb{R}^{n \times p}$ is the observer gain to be designed and the initial conditions $\underline{x}_{0}, \bar{x}_{0}$ are specified in Assumption 1 .

Theorem 1. Let assumptions 1, 2 be satisfied and $A-L C$ be a nonnegative and Schur stable matrix, then (5) is an IO for (1): the relation (2) is true and $\bar{x}_{k}-x_{k}, x_{k}-\underline{x}_{k} \in \mathcal{L}_{\infty}^{n}$.

Due to space limitations, all proofs are omitted.

The matrix $L$ can be computed by solving a system of LMIs [16]. However, we will present a tuning procedure more suitable to the problem investigated in this work in the next subsection.

Remark 1. The requirement that the matrix $A-L C$ has to be nonnegative can be relaxed using a proper change of coordinates as in [18]. For brevity of exposition, this extension will not be presented here.

In order to evaluate the interval precision of (5), let us consider the dynamics of the corresponding interval width $\delta x_{k}=\bar{x}_{k}-\underline{x}_{k}$ :

$$
\delta x_{k+1}=(A-L C) \delta x_{k}+\delta w_{k}+|L| \delta v_{k},
$$

where $\delta w_{k}=\bar{w}_{k}-\underline{w}_{k}$ and $\delta v_{k}=\bar{v}_{k}-\underline{v}_{k}$ determine the uncertainty size of the state and the output disturbances, respectively. Since $\delta x_{0}=\bar{x}_{0}-\underline{x}_{0}$, we obtain:

$\delta x_{k}=(A-L C)^{k}\left(\bar{x}_{0}-\underline{x}_{0}\right)+\sum_{i=0}^{k-1}(A-L C)^{k-1-i}\left(\delta w_{i}+|L| \delta v_{i}\right)$

for all $k \in \mathbb{Z}_{+}$. Hence, the values of $\delta x_{k}$ are completely determined by the choice of $L$ and the uncertainty levels given in assumptions 1 and 2 .

Denote

$$
\Delta_{k}=\left[-\frac{\delta x_{k}}{2}, \frac{\delta x_{k}}{2}\right], \quad \overline{\mathbb{X}}_{f}=\bigcup_{k \in \mathbb{Z}_{+}} \Delta_{k}
$$

as an admissible interval around the origin at each instant $k \in \mathbb{Z}_{+}$and the maximal terminal set, respectively. Roughly speaking, $\Delta_{k}$ provides the estimated interval for $x_{k} \simeq$ $\frac{\underline{x}_{k}+\bar{x}_{k}}{2} \simeq 0$ (i.e., when the control goal is reached), and $\overline{\mathbb{X}}_{f}$ covers all possible cases for $k \in \mathbb{Z}_{+}$. Another way of defining $\overline{\mathbb{X}}_{f}$ and $\Delta_{k}$ is to compute $\delta x_{k}$ under the hypothesis $\bar{x}_{0}=\underline{x}_{0}$, i.e., skipping the influence of initial condition uncertainty that anyway disappears asymptotically, and considering the asymptotic disturbance uncertainty only, then for $i_{0} \in \mathbb{Z}_{+}$ and $k \geq i_{0}$ :

$$
\delta x_{k}=\left\{\begin{array}{ll}
\sum_{i=i_{0}}^{k-1}(A-L C)^{k-1-i}\left(\delta w_{i}+|L| \delta v_{i}\right) & k \geq i_{0} \\
0 & k<i_{0}
\end{array} .\right.
$$

We need the following property, which is conventionally imposed in MPC [1]:

Assumption 3. Let the terminal set $\mathbb{X}_{f} \subseteq \overline{\mathbb{X}}_{f} \cap \mathbb{X}$.

The terminal set $\mathbb{X}_{f}$ represents the domain that has to be reached by a properly designed control sequence $u_{k}$ in a finite number of steps, and where a sub-optimal control strategy is activated in order to guarantee asymptotic stability and robustness for the closed loop system (see Section IV).

The hypothesis of Assumption 3 serves also as a necessary condition for the existence of a solution to the posed control problem (at least locally, in a vicinity of the origin) that is based on the estimates provided by the IO (5). Indeed, it means that if we start at a neighborhood of the origin and assumptions 1, 2 are satisfied, then obtained interval estimates $\left[\underline{x}_{k}, \bar{x}_{k}\right]$ belong to $\mathbb{X}_{f} \subseteq \mathbb{X}$ provided that the control $u_{k}$ is designed such that the interval center $x_{k}^{\star}=\frac{x_{k}+\bar{x}_{k}}{2}$ (and, hence, $x_{k}$ ) is kept close to the origin.

\section{B. Predictor}

As readily seen, IO (5) calculates an interval of admissible values $\left[\underline{x}_{k}, \bar{x}_{k}\right]$ of the state $x_{k}$ for all $k \in \mathbb{Z}_{+}$. Hence, this information can be easily used to check the state constraints:

$$
\left[\underline{x}_{k}, \bar{x}_{k}\right] \subset \mathbb{X} \Rightarrow x_{k} \in \mathbb{X},
$$

but the equations (5) depend on an output injection term $L y_{k}$, which is unknown in future steps, making it unsuitable for prediction of the system behavior (required for application of the MPC methodology). However, we can design an IP that satisfies (2) utilizing solely information on the system dynamics and bounds of the disturbance $w_{k}$ under the following mild hypothesis (which can always be verified by a suitable change of coordinates):

Assumption 4. Let $C \geq 0$.

By definition $L=L^{+}-L^{-}$, where $L^{+}, L^{-} \in \mathbb{R}_{+}^{n \times p}$, then under Assumption 4, (2) and according to (3):

$$
L^{+} C \underline{x}_{k}-L^{-} C \bar{x}_{k} \leq L C x_{k} \leq L^{+} C \bar{x}_{k}-L^{-} C \underline{x}_{k},
$$

which allows us to replace in (4) the uncertain for prediction terms $L y_{k}-L v_{k}+w_{k}=L x_{k}+w_{k}$ with their bounds to obtain an IP:

$$
\begin{aligned}
& \bar{z}_{k+1}=(A-L C) \bar{z}_{k}+B u_{k}+L^{+} C \bar{z}_{k}-L^{-} C \underline{z}_{k}+\bar{w}_{k} \\
& \underline{z}_{k+1}=(A-L C) \underline{z}_{k}+B u_{k}+L^{+} C \underline{z}_{k}-L^{-} C \bar{z}_{k}+\underline{w}_{k}
\end{aligned},
$$

where $\bar{z}_{k} \in \mathbb{R}^{n}$ and $\underline{z}_{k} \in \mathbb{R}^{n}$ are the upper and the lower predicted bounds for $x_{k}, k \in \mathbb{Z}_{+}$, respectively. As we can conclude, (7) is composed only of known terms under assumptions 1 and 2 , and also of $u_{k}$ to be computed in the control procedure.

Remark 2. Note that different gains $L$ can be used in the IO (5) and in the IP (7). This may lead to a less restrictive estimation result, but to streamline the presentation such an extension is left for a future research.

Introducing a change of coordinates to new variables $z_{k}^{\star}=$ $\frac{\bar{z}_{k}+\underline{z}_{k}}{2}$ (the center of the interval) and $\delta z_{k}=\bar{z}_{k}-\underline{z}_{k}$ (the amplitude of the interval), one gets the following equivalent representation of dynamics of (7):

$$
\begin{aligned}
z_{k+1}^{\star} & =A z_{k}^{\star}+B u_{k}+w_{k}^{\star}, \\
\delta z_{k+1} & =\left(A+2 L^{-} C\right) \delta z_{k}+\delta w_{k}
\end{aligned}
$$


where, similarly, $w_{k}^{\star}=\frac{\bar{w}_{k}+\underline{w}_{k}}{2}$ and $\delta w_{k}=\bar{w}_{k}-\underline{w}_{k}$. The dynamics of the center of the predicted interval $z_{k}^{\star}$ is independent on $L$, but it is controlled by $u_{k}$, hence, (8) can be used in MPC. The dynamics of the interval width $\delta z_{k}$ is governed by the interval width of the state disturbance $\delta w_{k}$, and it has to be optimized by a choice of $L$. This brings us to the following result:

Theorem 2. Let assumptions 1, 2, 4 be satisfied, and there exist a diagonal matrix $P \in \mathbb{R}^{n \times n}$, matrices $Q, \Gamma \in \mathbb{R}^{n \times n}$ and $U^{-}, U^{+} \in \mathbb{R}_{+}^{n \times p}$ such that the following linear matrix inequalities are verified:

$$
\left[\begin{array}{ccc}
P A-U^{+} C+U^{-} C \geq 0, \\
P-Q & 0 & A^{\top} P+2 C^{\top} U^{-\top} \\
0 & \Gamma & P \\
P A+2 U^{-} C & P & P
\end{array}\right] \succeq 0
$$

Then for the system (1), the IP (7) with $L^{-}=P^{-1} U^{-}$, $L^{+}=P^{-1} U^{+}$and $\underline{z}_{0}=\underline{x}_{0}, \bar{z}_{0}=\bar{x}_{0}$ satisfies the relation:

$$
\underline{z}_{k} \leq x_{k} \leq \bar{z}_{k} \quad \forall k \geq 0,
$$

and $\delta z \in \mathcal{L}_{\infty}^{n}$.

Note that since $0 \leq A-L C \leq A+2 L^{-} C$, restraining $A+2 L^{-} C$ to be Schur stable (as imposed by Theorem 2) automatically implies that $A-L C$ is also Schur stable, fulfilling the conditions of Theorem 1 .

\section{Comparison of IO and IP}

It is important to highlight that the inclusion $\left[\underline{x}_{k}, \bar{x}_{k}\right] \subseteq$ $\left[\underline{z}_{k}, \bar{z}_{k}\right]$ is not necessary valid for all $k>0$. Indeed, to compare the interval estimation accuracy of (5) and (7) (under assumption that $z_{k}^{\star} \simeq x_{k}^{\star}$ ), let us introduce the relative error $\delta \xi_{k}=\delta z_{k}-\delta x_{k}$, whose increment according to (6) and (9) takes the form

$$
\delta \xi_{k+1}=(A-L C) \delta \xi_{k}+|L|\left(C \delta z_{k}-\delta v_{k}\right) .
$$

Proposition 1. If conditions of Theorem 2 are satisfied, then $\left[\underline{x}_{k}, \bar{x}_{k}\right] \subseteq\left[\underline{z}_{k}, \bar{z}_{k}\right]$ for all $k \in \mathbb{Z}_{+}$in (5) and (7) provided that $z_{k}^{\star}=x_{k}^{\star}$ and

$$
C \delta z_{k} \geq \delta v_{k} \quad \forall k \in \mathbb{Z}_{+} .
$$

Hence, in general for a sufficiently big measurement noise (big $\delta v_{k}$ ) the property $\left[\underline{x}_{k}, \bar{x}_{k}\right] \subseteq\left[\underline{z}_{k}, \bar{z}_{k}\right]$ may be failed. The condition (11) admits also the following interpretation: if the width of interval prediction for the output variable $C \delta z_{k}$ is bigger than the measurement perturbation uncertainty $\delta v_{k}$, then an IO has a better accuracy of estimation than an IP.

Remark 3. Under conditions of Theorem 2, the property (11) can be verified "off-line" since for its computation we do not need measurements $y_{k}$ (only information given in assumptions 1,2 is needed).

\section{Feedback stabilization of IP}

Note that IP (7) can be stabilized by designing a feedback for its completely known center dynamics (8) as follows:

$$
u_{k}=S_{f} w_{k}^{\star}+K_{f} z_{k}^{\star}
$$

with the gains $K_{f} \in \mathbb{R}^{m \times n}$ and $S_{f} \in \mathbb{R}^{m \times n}$, which guarantee asymptotic stability of the closed-loop system and minimization the influence of $w_{k}^{\star}$, respectively. These gains can be selected as follows:

Proposition 2. Let $S_{f}=\Sigma P^{-1}$ and $K_{f}=\Upsilon P^{-1}$, where $P \in \mathbb{R}^{n \times n}$ and $\Sigma, \Upsilon \in \mathbb{R}^{m \times n}$ are solutions of a linear optimization problem:

$$
\begin{aligned}
& \max _{Q, \Gamma \in \mathbb{R}^{n \times n}} Q-\Gamma, \\
& Q=Q^{\top} \succ 0, \Gamma=\Gamma^{\top} \succ 0, P=P^{\top} \succ 0, \Pi \succeq 0 \\
& \Pi=\left[\begin{array}{cccc}
P-Q & P+B \Sigma & P A^{\top}+\Upsilon^{\top} B^{\top} & 0 \\
P+\Sigma^{\top} B^{\top} & \Gamma & 0 & P+\Sigma^{\top} B^{\top} \\
A P+B \Upsilon & 0 & P & 0 \\
0 & P+B \Sigma & 0 & P
\end{array}\right] .
\end{aligned}
$$

Then the center dynamics (8) with the control (12) is ISS (from the input $w_{k}^{\star}$ to the state $z_{k}^{\star}$ ) with the optimal attenuation of the known disturbance $w_{k}^{\star}$ and with an ISS-Lyapunov function $V\left(z_{k}^{\star}\right)=z_{k}^{\star \top} P^{-1} z_{k}^{\star}$.

If $w_{k}^{\star}=0$ for $k \in \mathbb{Z}_{+}$, then an obvious choice is $S_{f}=$ 0 (a constraint to impose for the optimization problem in Proposition 2).

\section{IO-MPC DESIGN}

This section presents the output-feedback MPC scheme with guaranteed robust constraint satisfaction based on IO (5) and IP (7) given in the previous section.

As framer (7) depends solely on known variables, it can be used for prediction in a receding horizon fashion. Indeed, the central trajectory of the interval containing $x_{k}$ modeled by (8) is controlled by $u_{k}$, while the width in (9) is stable, provided that the conditions of Theorem 2 are satisfied.

The principal steps are as follows:

1) The IO (5) evaluates an interval of admissible values $\left[\underline{x}_{k}, \bar{x}_{k}\right]$ for $x_{k}$ with $k \in \mathbb{Z}_{+}$. Under Assumption 3, there exists $k_{0} \in \mathbb{Z}_{+}$such that $\left[\underline{x}_{k}, \bar{x}_{k}\right] \cap \mathbb{X}_{f} \neq \emptyset$ for all $k \geq k_{0}$ provided that the interval center $x_{k}^{\star}=\frac{\underline{x}_{k}+\bar{x}_{k}}{2}=$ 0 , which has to be ensured by the control $u_{k}$ design.

2) At each $k \in \mathbb{Z}_{+}$, the IP (7) is initialized as $\underline{z}_{k, 0}=$ $\underline{x}_{k}$ and $\bar{z}_{k, 0}=\bar{x}_{k}$ (i.e., by taking the interval derived in (5) using the measurements $y_{k}$ and the inputs $u_{k}$ ), and having a sequence of inputs $\mathcal{S}_{N}=\left\{s_{0}, \ldots, s_{N-1}\right\}$ with $s_{i} \in \mathbb{U}$ for all $i=0, \ldots, N-1$, where $N>1$ is the length of the prediction horizon, we will calculate the values $\underline{z}_{k, i+1}, \bar{z}_{k, i+1}$ for $i=0, \ldots, N-1$ under substitution $u_{k+i}=s_{i}$ (it is supposed in Assumption 2 that $\underline{w}_{k}$ and $\bar{w}_{k}$ are given for all $\left.k \in \mathbb{Z}_{+}\right)$; and $\delta z_{k, i}=$ $\bar{z}_{k, i}-\underline{z}_{k, i}$ whose dynamics are governed by (8) and (9), respectively. 
3) The sequence $\mathcal{S}_{N}$ will be introduced through solution of the optimization problem posed for (8):

$$
\mathcal{S}_{N}^{k}:=\arg \min _{\mathcal{S}_{N}} V_{N}\left(z_{k, 0}^{\star}, \ldots, z_{k, N}^{\star}, \mathcal{S}_{N}\right)
$$

subject to the following constraints

$$
\begin{aligned}
& \underline{z}_{k, i+1}, \bar{z}_{k, i+1} \text { are computed by (7), } \\
& \underline{z}_{k, 0}=\underline{x}_{k}, \quad \bar{z}_{k, 0}=\bar{x}_{k}, \\
& {\left[\underline{z}_{k, i+1}, \bar{z}_{k, i+1}\right] \subset \mathbb{X}, \quad s_{i} \in \mathbb{U},} \\
& z_{k, N}^{\star} \in \mathbb{X}_{f},
\end{aligned}
$$

where $V_{N}\left(z_{k, 0}^{\star}, \ldots, z_{k, N}^{\star}, \mathcal{S}_{N}\right)=V_{f}\left(z_{k, N}^{\star}\right)+$ $\sum_{i=0}^{N-1} \ell\left(z_{k, i}^{\star}, s_{i}\right)$ with $V_{f}(z)=z^{\top} W z, \quad \ell(z, s)=$ $z^{\top} H z+s^{\top} R s$, and the weighting matrices $W, H \in \mathbb{R}^{n \times n}$ and $R \in \mathbb{R}^{m \times m}$ are positive definite and symmetric.

The feedforward control is then selected as

$$
u_{k}=s_{0}^{k} .
$$

In order to guarantee proper asymptotically behaviour of the closed-loop system, we use the idea of dual mode formulation [1]. Basically, the open-loop control (15) is used to steer the system to the terminal set $\mathbb{X}_{f} \subset \mathbb{X}$, where a local (static) controller (12) under substitution $z_{k}^{\star}=x_{k}^{\star}$ can be applied (the gains $K_{f} \in \mathbb{R}^{m \times n}$ and $S_{f} \in \mathbb{R}^{m \times n}$ fulfill the conditions of Proposition 2). As usual in MPC [1], we will require the following auxiliary properties for the feedback gains $S_{f}$ and $K_{f}$ (denote $\mathbb{W}=\bigcup_{k \in \mathbb{Z}_{+}} w_{k}^{\star}$ and $\left.\mathbb{V}=\bigcup_{k \in \mathbb{Z}_{+}}\left[-\frac{\delta v_{k}}{2}, \frac{\delta v_{k}}{2}\right]\right)$ :

Assumption 5. For any $x \in \mathbb{X}_{f}, w \in \mathbb{W}$ and $\eta \in \overline{\mathbb{X}}_{f}+\mathbb{V}$ :

$$
\begin{gathered}
\left(A+B K_{f}\right) x+\left(I_{n}+B S_{f}\right) w+L \eta \in \mathbb{X}_{f}, \\
S_{f} w+K_{f} x \in \mathbb{U} .
\end{gathered}
$$

These restrictions imply that the feedback (12) ensures the forward invariance of $\mathbb{X}_{f}$ and satisfies the constraints on control. Therefore, similarly to [19], the control law for (1) will be selected as a combination of (15) and (12):

$$
u_{k}=\left\{\begin{array}{ll}
s_{0}^{k} & x_{k}^{\star} \notin \mathbb{X}_{f} \\
S_{f} w_{k}^{\star}+K_{f} x_{k}^{\star} & x_{k}^{\star} \in \mathbb{X}_{f}
\end{array} .\right.
$$

Now we are in position to formulate the main result of this section:

Theorem 3. Let $\left[\underline{x}_{0}, \bar{x}_{0}\right] \subset \mathbb{X},(11)$ and assumptions 1-5 hold with $\bar{w}_{k}, \underline{w}_{k}=$ const for all $k \in \mathbb{Z}_{+}$. Then the closed-loop system given by (1), (5) and (16) has the following properties:

1) Recursive feasibility with reaching $\mathbb{X}_{f}$ in $N$ steps;

2) ISS for the dynamics of $x_{k}^{\star}$ in the terminal set $\mathbb{X}_{f}$;

3) Constraint satisfaction.

Proof. The proof relies on conventional arguments for MPC schemes (see [1]) and is omitted due to space limitations.

\section{Numerical ExAmple}

In this section, we illustrate the usefulness of the presented methodology through a numerical example. A comparison with classical results shown in [5], [6] is also performed. The example considered is the continuous stirred tank reactor (CSTR), where an exothermic and irreversible reaction $S \rightarrow$ $P$ occurs.

The model (see [20]) is composed by two states, $C_{s}$ and $T$, the concentration of $S$ and the temperature of the reactor, respectively. The controlled input is the coolant stream $T_{C}$. The linearization of this model around a stable equilibrium point, discretized with a sampling rate $\tau=0.25 \mathrm{~min}$, results in

$$
A=\left[\begin{array}{cc}
0.745 & -0.002 \\
5.610 & 0.780
\end{array}\right], B=\left[\begin{array}{c}
5.6 \times 10^{-6} \\
0.464
\end{array}\right], C=\left[\begin{array}{ll}
0 & 1
\end{array}\right]
$$

For simulation purposes, the initial conditions are $x_{0}=$ $[0,0]$. The constraint sets are considered as $\mathbb{X}=[-0.4,0.4] \times$ $[-25,25]$ and $\mathbb{U}=[-15,15]$, while the disturbances are restricted to $\mathbb{W}=[-0.02,0.02] \times[-0.4,0.4]$ and $\mathbb{V}=$ $[-0.1,0.1]$.

In accordance with Section III, the IO (5) and the IP (7) are designed following the discussion presented in Theorem 2, which the gains $L=\left[\begin{array}{ll}-0.002 & 0.390\end{array}\right]^{\top}$ and $L^{-}=$ $\left[\begin{array}{ll}0.002 & 0\end{array}\right]^{\top}$. The IO and the IP are then initialized with $\underline{z}_{0}=\underline{x}_{0}=[-0.1,-0.05]$ and $\bar{z}_{0}=\bar{x}_{0}=[0.1,0.05]$. This fulfills Assumption 2.

The MPC is designed to track a set-point $x_{r}=$ $[-0.25,27.3])$, hence the cost function is modified to include an error term $x-x_{r}$ (for set-point tracking - although feasible for system (1), this point is outside the constraint set). The selected matrices are $H=100 I_{2}$ and $R=0.01$ and the prediction horizon is $N=10$. The terminal cost, $W$, is selected by computing the associated LQR controller (this is possible due to the discussion after Proposition 2).

In the following, Figs. 1 and 2 illustrate the evolution of the states using the IO-MPC and Tube-MPC, respectively. Both simulations consider several realizations of disturbances $w_{k}$ and $v_{k}$. As it can be seen, our approach steers the states very close to the boundaries of the constraint set, indicating very low conservativeness, in opposition to the same scenario but using the Tube method from [5], [6].

The advantages of our method are its reduced complexity and its constructiveness. The computational complexity is similar to nominal MPC and, also, no optimization of initial conditions is needed (compare [5], [6]). Furthermore, in Tube-MPC, the controller/observer gains must be carefully chosen, since it affects directly the computation of the uncertainty sets. In our approach, all gains are determined constructively (given as LMIs).

Indeed, using the IP alleviates the complexity of uncertainty propagation, since it is constantly updated by inputoutput information from the IO, which also provides a tighter membership interval for the uncertain trajectories.

All simulations have been performed in MATLAB 2017a, using an Intel i7-8565U processor $(1.8 \mathrm{GHz})$ and 16GB RAM. 
Also, toolboxes such as MPT3 [21] and PnPMPC [22] were used.

Remark 4. It is worth noticing that our approach does not require any steady-state assumption for the observer nor any further development for compensating the initial uncertainty (compare [3], [6]), since these features are automatically attained by the convergence of the pair IO/IP.
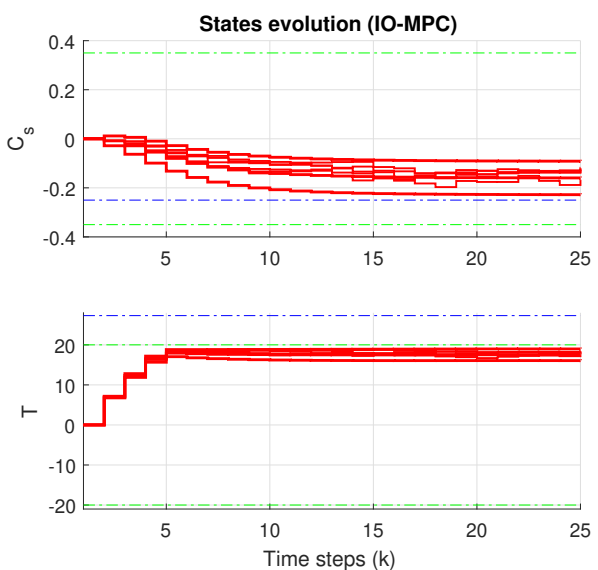

Figure 1. Results obtained with IO-MPC. Legend: green lines: boundaries of the constraint set, blue lines: set-points to be tracked, and red lines: evolution of each state (considering several extremal realizations of $w_{k}$ and $v_{k}$ ).
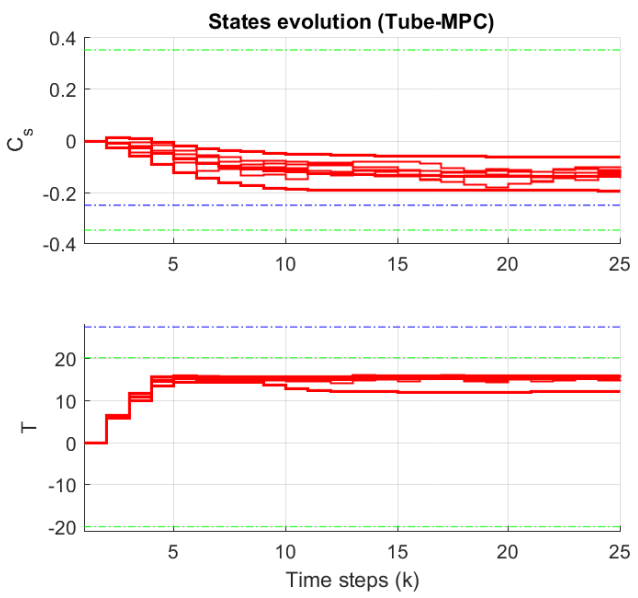

Figure 2. Results obtained with Tube-MPC. The legend is the same as for the previous figure.

\section{CONCLUSION}

In this paper, we presented a novel robust output-feedback MPC using interval observers and predictors. Utilizing the simple form of such estimators, robust constraint satisfaction is achieved with reduced computational complexity. The efficiency of presented results is illustrated by numerical simulation of a chemical reactor in comparison with a conventional solution.
This new approach offers interesting directions for future research on output-feedback MPC schemes, such as extensions to linear parameter-varying (LPV), time-delayed and some classes of nonlinear systems.

\section{REFERENCES}

[1] D. Q. Mayne, J. B. Rawlings, C. V. Rao, and P. O. M. Scokaert, "Constrained model predictive control: stability and optimality," Automatica, vol. 36, pp. 789-814, 2000.

[2] A. Bemporad and M. Morari, "Robust model predictive control: A survey," in Robustness in Identification and Control, 1998.

[3] A. Bemporad and A. Garulli, "Output-feedback predictive control of constrained linear systems via set-membership state estimation," International Journal of Control, vol. 73, no. 8, pp. 655-665, 2000.

[4] A. Bemporad, F. Borrelli, and M. Morari, "Min-max control of constrained uncertain discrete-time linear systems," IEEE Transactions on Automatic Control, vol. 48, no. 9, pp. 1600-1606, 2003.

[5] D. Q. Mayne, S. V. Ravokic, R. Findeisen, and F. Allgower, "Robust output feedback model predictive control of constrained linear systems," Automatica, vol. 42, pp. 1217-1222, 2006.

[6] _ _ "Robust output feedback model predictive control of constrained linear systems: time-varying case," Automatica, vol. 45, pp. 20822087, 2009.

[7] J. Gouzé, A. Rapaport, and M. Hadj-Sadok, "Interval observers for uncertain biological systems," Ecological Modelling, vol. 133, no. 1, pp. 45-56, 2000.

[8] F. Mazenc and O. Bernard, "Interval observers for linear time-invariant systems with disturbances," Automatica, vol. 47, no. 1, pp. 140-147, 2011.

[9] S. Chebotarev, D. Efimov, T. Raïssi, and A. Zolghadri, "Interval observers for continuous-time LPV systems with $\ell_{1} / \ell_{2}$ performance," Automatica, 2015.

[10] T. Raïssi, D. Efimov, and A. Zolghadri, "Interval state estimation for a class of nonlinear systems," IEEE Trans. Automatic Control, vol. 57, no. 1, pp. 260-265, 2012

[11] D. Efimov, W. Perruquetti, T. Raïssi, and A. Zolghadri, "Interval observers for time-varying discrete-time systems," IEEE Trans. Automatic Control, vol. 58, no. 12, pp. 3218-3224, 2013

[12] D. Efimov, W. Perruquetti, and J.-P. Richard, "Interval estimation for uncertain systems with time-varying delays," International Journal of Control, vol. 86, no. 10, pp. 1777-1787, 2013.

[13] T. N. Dinh, F. Mazenc, and S.-I. Niculescu, "Interval observer composed of observers for nonlinear systems," European Control Conference, 2014.

[14] E. Leurent, D. Efimov, T. Raïssi, and W. Perruquetti, "Interval prediction for continuous-time systems with parametric uncertainties," in Proc. 58th IEEE Conference on Decision and Control (CDC), Nice, 2019.

[15] D. Efimov and T. Raïssi, "Design of interval observers for uncertain dynamical systems," Autom. Remote Control, vol. 77, no. 2, pp. 191225, Feb. 2016.

[16] D. Efimov, W. Perruquetti, T. Raïssi, and A. Zolghadri, "On interval observer design for time-invariant discrete-time systems," in 2013 European Control Conference (ECC), 2013, pp. 2651-2656.

[17] L. Farina and S. Rinaldi, Positive Linear Systems: Theory and Applications. John Wiley \& Sons, 2000.

[18] F. Mazenc, T. N. Dinh, and S. I. Niculescu, "Interval observers for discrete-time systems," International Journal of Robust and Nonlinear Control, vol. 24, pp. 2867-2890, 2014.

[19] H. Michalska and D. Q. Mayne, "Robust receding horizon control of constrained nonlinear systems," IEEE Transactions on Automatic Control, vol. 38, no. 11, pp. 1623-1633, 1993.

[20] M. A. Henson and D. E. Seborg, Eds., Nonlinear Process Control. USA: Prentice-Hall, Inc., 1997.

[21] M. Herceg, M. Kvasnica, C. Jones, and M. Morari, "MultiParametric Toolbox 3.0," in Proc. European Control Conference (ECC), Zürich, Switzerland, July 17-19 2013, pp. 502-510, http://control.ee.ethz.ch/ mpt.

[22] S. Riverso, A. Battocchio, and G. Ferrari-Trecate, "Pnpmpc toolbox," 2013. [Online]. Available: http://sisdin.unipv.it/pnpmpc/pnpmpc.php

[23] Z.-P. Jiang and Y. Wang, "Input-to-state stability for discrete-time nonlinear systems," Automatica, vol. 37, no. 6, pp. 857-869, 2001. 